\title{
The where's and when's of kinase anchoring
}

\section{F. Donelson Smith, Lorene K. Langeberg and John D. Scott}

Howard Hughes Medical Institute, Vollum Institute, Oregon Health and Sciences University, MRB322, 3181 SW Sam Jackson Park Road, Portland, OR 97239, USA

Kinase anchoring has gained acceptance as a means to synchronize spatial and temporal aspects of cell signaling. A-kinase anchoring proteins (AKAPs) are a diverse group of functionally related proteins that target protein kinase $A$ and other enzymes to coordinate a range of signaling events. Recent advances in this field have shown that incorporating phosphodiesterases into AKAP signaling complexes exerts local control of cAMP metabolism, that phosphorylation of some AKAPs potentiates downstream signaling events, that anchoring of distinct enzyme combinations functions as a mechanism to expand the repertoire of cellular events controlled by a single AKAP, and that fluorescent biosensors can be used to visualize dynamic aspects of localized cAMP signaling.

\section{Introduction}

A half-century of work has proclaimed protein phosphorylation as a principal means of reversibly controlling biochemical events. It began when Fischer and Krebs [1] demonstrated that conversion of inactive muscle phosphorylase $b$ into active phosphorylase $a$ requires ATP and is catalyzed by a phosphorylase $b$ kinase. They then showed that phosphorylase $b$ kinase itself is controlled by a serine kinase that is responsive to the second messenger cAMP. Together with their colleagues, they subsequently discovered protein kinase A (PKA) and the concept of a 'kinase cascade' [2].

From these humble beginnings the field of protein kinase research was born, paving the way for the discovery of phosphotyrosine by Hunter and co-workers [3], the identification of Src tyrosine kinase by Brugge and Ericsson et al. [4], the seminal work of Pawson and associates on phosphotyrosine recognition motifs [5], and the resolution of a crystal structure of the PKA catalytic subunit by Taylor and colleagues [6]. Consequently, we now recognize that the $\sim 518$ members of the human kinome represent a superfamily of enzymes that participate in all aspects of cellular regulation [7].

Protein kinase research is now focusing on how these enzymes are organized in relation to their effectors and substrates within the three dimensions of the cell. Interestingly, the study of PKA is shedding new light on the complexity of these protein-protein interactions.

Corresponding author: Scott, J.D. (scott@ohsu.edu).

Available online 11 May 2006
A-kinase anchoring proteins (AKAPs) have emerged as important signal-organizing components for PKA and other kinases. Here, we discuss their role in the spatiotemporal control of cAMP signaling and other transduction events.

\section{In the beginning*}

It was clear from early physiology experiments that stimulation of cAMP synthesis by different agonists produces distinct physiological outputs, even within the same tissue. It was subsequently suggested that the occupancy of particular G-protein-coupled receptors (GPCRs) favored the activation of PKA pools located in different subcellular compartments. Conclusive evidence supporting this concept was not obtained until the early 1980 s, however, when it was shown that adrenergic stimulation selectively activates a pool of PKA associated with the particulate fraction of cardiomyocytes, whereas stimulation with prostaglandin $\mathrm{E}_{1}$, which induces different physiological effects, activates a cytosolic pool of PKA in the same cells [8]. These findings were consistent with earlier biochemical evidence showing that the PKA holoenzyme, which comprises two regulatory subunits and two catalytic subunits, exists in two forms: a type I PKA holoenzyme, which was then thought to be cytoplasmic; and a type II PKA holoenzyme, which was considered to be exclusively particulate. There was no evidence, however, to indicate how the two PKA holoenzymes were retained in different subcellular compartments.

The vital link was provided in 1982 by Theurkauf and Vallee [9], who demonstrated that type II PKA co-purifies with microtubules and that its regulatory RII subunit binds to the microtubule-associated protein MAP2 [9]. Thus, the first AKAP was identified. Many more of these RII-binding proteins were detected when Lohmann et al. [10] developed an overlay technique to probe proteins immobilized on nitrocellulose membranes with the purified RII subunit. By the late 1980s, Rubin, Erlichman and co-workers $[11,12]$ had used this technology to characterize a bovine brain protein, called P75, and its murine ortholog, $\mathrm{P} 150$ (these proteins are now known as AKAP75 (human AKAP79) and AKAP150, respectively; see Box 1 for AKAP naming conventions).

\footnotetext{
This section is inspired by a historical outline of the founding of the AKAP field delivered in a lecture by J.D.S. at the First International Meeting on Anchored cAMP Signalling Pathways held in Berlin, October 15-16, 2005.
} 


\section{Box 1. What's in a name?}

Originally AKAPs were named according to their apparent molecular mass determined by SDS polyacrylamide gel electrophoresis or by prediction from the open reading frame: AKAP79, for example, migrates at $\sim 79 \mathrm{kDa}$ on a gel. Several AKAPs, such as the muscleselective mAKAP (also known as AKAP100) and AKAP-Lbc, were subsequently found to be fragments or smaller transcripts of larger genes and were renamed. More recently, proteins such as Gravin, Ezrin, Rab32 and WAVE-1 have been identified as AKAPs but retain their original designation.

With the sequencing and annotation of several genomes has arisen a new nomenclature in nucleotide and protein databases in which AKAPs are numbered sequentially with no regard to their order of discovery or reference to their traditional name. Added confusion is introduced because the database curators have not included AKAPs such as Ezrin, Rab32 and WAVE-1 in their classification. Although we understand the necessity for a standard nomenclature, we have noted several recent examples in which an individual AKAP, such as AKAP79, is simply referred to as 'AKAP'. This nomenclature is not correct and leads to confusion regarding the anchoring protein that is actually being studied. We therefore recommend the continued use of the original AKAP nomenclature. See Tasken and Aandahl [27] for a comprehensive table of AKAPs.

Armed with the overlay assay, our laboratory (http:// www.ohsu.edu/vollum/faculty/scott) entered this field in the early 1990 s, showing that the RII subunit must be dimerized for interaction with AKAPs and that each anchoring protein contains a reciprocal binding sequence of 14-18 amino acids that form an amphipathic helix $[13,14]$. The RII overlay assay was adapted to screen phage cDNA libraries and has been used to clone several novel AKAPs [15]. One of these proteins, initially called Ht31 but now known as AKAP-Lbc, contains a sequence of 18 amino acids that has been used as a peptide disruptor of RII-AKAP interactions inside cells [16,17]. The utility of this peptide as a disruptor of PKA anchoring was first demonstrated when we were involved in studies showing that perfusion of Ht31 into cultured hippocampal neurons disrupts the location of PKA in relation to a key substrate, the $\alpha$-amino-3-hydroxy-5-methyl-4-isoxazole propionic acid (AMPA)-type glutamate receptor [18]. The functional consequence of this disruption was to decrease the responsiveness of the ion channel to synaptic signals [18]. At the same time, Caterall's group [19] used the Ht31 peptide to demonstrate that disruption of PKA anchoring uncouples cAMP-dependent regulation of the L-type $\mathrm{Ca}^{2+}$ channel.

In 1997, a cell-soluble version of Ht31 was generated by conjugating a steroyl group to the $\mathrm{N}$ terminus [20]. This peptide and other Ht31 derivatives are now widely used to establish whether anchored pools of PKA participate in various cAMP signaling events [21,22]. Elegant structural studies from Jennings and colleagues $[23,24]$ have shown that a hydrophobic face on Ht31 fits into a groove created by the N-terminal regions of the RII dimer, forming a nanomolar-affinity complex. Related work from Taylor's group $[25,26]$ has shown that a subset of dual-specificity AKAPs can also interact with the type RI subunit. Thus, by the late $1990 \mathrm{~s}$, two important biochemical properties of AKAPs were emerging: first, AKAPs bind the R subunit dimer through a well-conserved amphipathic $\alpha$-helical motif; and second, each anchoring protein is targeted to a unique localization in a given cell type by an identifiable targeting motif [27].

\section{AKAP signaling complexes}

Another biological role of AKAPs became apparent when it was discovered that AKAP79 (the human ortholog of AKAP75 and AKAP150) not only anchored PKA but also bound the protein phosphatase PP2B [28]. This finding changed the way in which we think about AKAPs because it suggested that signals controlling phosphorylation and dephosphorylation of a single substrate can pass through the same AKAP signaling complex.

The notion of multivalent AKAPs continued to evolve when subsequent studies showed that AKAP79 also binds protein kinase $\mathrm{C}$ (PKC), thereby providing a means to integrate cAMP with $\mathrm{Ca}^{2+}$ and phospholipid signals at the same subcellular locus [29]. We now know that Gravin (AKAP250) and AKAP-Lbc colocalize PKA with PKC, whereas AKAP220 and AKAP149 place PKA in close proximity to the type 1 protein phosphatase PP1 [30-33]. In fact, there is reason to believe that most, if not all, anchoring proteins bring PKA together with other protein kinases, protein phosphatases and various signal termination enzymes. In the following sections, we discuss the role of these AKAP signaling complexes in the synchronization of compartmentalized signal transduction events.

\section{AKAPs and phosphodiesterases: compartmentalization of cAMP action}

Spatiotemporal control of cAMP flux requires the concerted action of two enzyme classes: adenylyl cyclases, which synthesize cAMP; and compartmentalized pools of phosphodiesterases (PDEs), which locally metabolize cAMP into 5'-AMP. The laboratories of Houslay (http:// www.gla.ac.uk/ibls/BMB/mdh) and Conti (http://wwwmed.stanford.edu/profiles/Marco_Conti) have identified several PDE-binding proteins that target distinct isozymes to specific cellular microenvironments [34]. A prototypical example is PDE4D3, which is differentially localized via interactions with scaffolding proteins such as myomegalin and $\beta$-arrestin [35-37]. Likewise, many AKAPs cluster PKA with PDEs to terminate cAMP signals as they diffuse into the cell [38,39].

In cardiomyocytes, the muscle-selective AKAP (mAKAP) assembles a negative feedback loop containing PKA and PDE4D3 [38] (Figure 1a). PKA-mediated phosphorylation of Ser13 in PDE4D3 augments mAKAP binding [40], whereas phosphorylation of Ser54 enhances the catalytic efficiency of the enzyme to favor cAMP metabolism [41]. These effects are counterbalanced by extracellular-signal-regulated kinases (ERKs) that phosphorylate PDE4D3 on Ser579 to suppress PDE activity [42]. This latter phosphorylation event might be catalyzed by ERK5, which is also a component of the mAKAP complex $[43,44]$. This configuration not only ensures bidirectional control of PDE4D3 activity but also has been postulated to generate local fluctuations in cAMP and concomitant pulses of PKA activity (Figure 1a).

Work from Tasken et al. [45] suggests that AKAP450 performs an analogous function at centrosomes, where it organizes a signaling complex of PKA, PDE4D3 and 
(a)

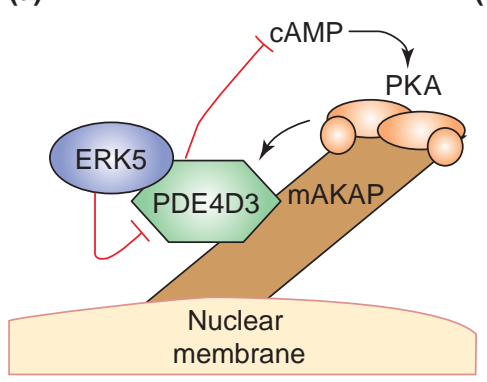

(b)

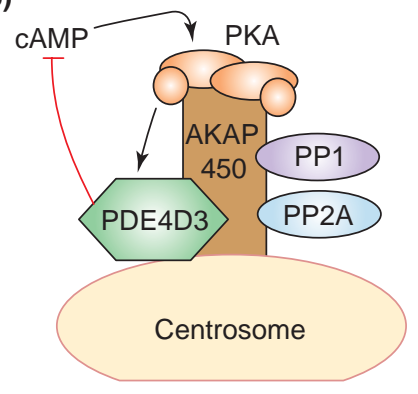

(c)

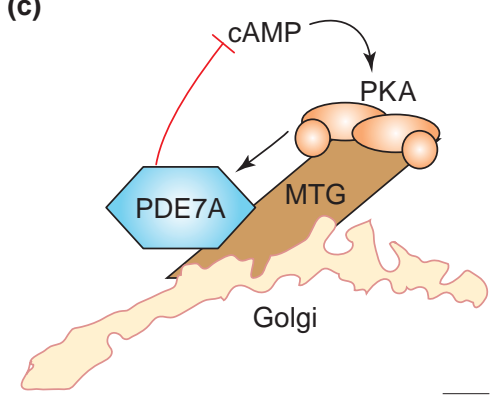

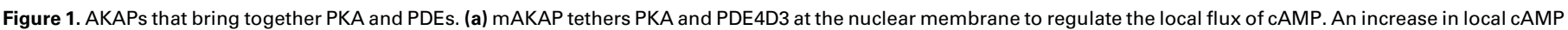

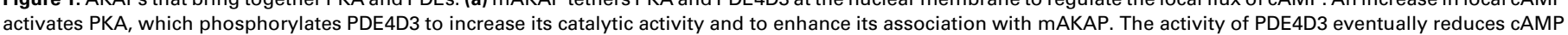
back to basal concentrations and shuts off the kinase. PDE4D3 also acts as a scaffold to bring ERK5 (and its upstream activating kinase MEK5) into the complex. ERK5 negatively regulates PDE4D3 activity by phosphorylating it on distinct sites. This arrangement enables mAKAP and PDE4D3 to act as integrators of cAMP and mitogenic

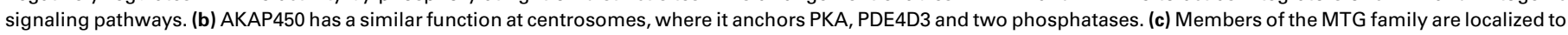
the Golgi, where they target PKA and the PDE7A isoform.

the protein phosphatases PP1 and PP2A (Figure 1b). Likewise, AKAP110 targets the PDE4A isoform to the acrosome of sperm, whereas AKAP149 and AKAP121 and the Nervy and myeloid translocation gene (MTG) anchoring proteins, respectively, target the PDE4A and PDE7A isoforms to various subcellular locations [46,47] (Figure 1c). These AKAP-PKA-PDE units respond to upstream signals that emanate from GPCR and adenylyl cyclase networks, creating a complex and continually changing signaling environment in which cAMP concentrations are distributed unevenly in the cell. These findings underscore the pleiotropic nature of this important second messenger and highlight the central role of AKAPs in the customized regulation of cAMP signaling.

\section{Phosphorylation in AKAP complexes}

Soon after AKAPs were discovered, it was postulated that they that would be the preferred substrates for their associated kinases [48]. Although experimental evidence supporting this prediction has been slow in coming, three recent examples suggest that it might be correct.

First, the AKAP Yotiao associates with various ion channels, including the KCNQ1 subunit of $\mathrm{K}^{+}$channels responsible for $I_{\mathrm{KS}}$ currents that shape the duration of cardiac action potentials in response to $\beta$-adrenergic agonists [49] (Figure 2a). Inherited mutations in KCNQ1 subunits have been linked to long QT syndrome (LQTS), a disease characterized by cardiac arrhythmias and sudden death [50]. Intriguing work from Kass and co-workers $[51,52]$ shows that the anchored PKA phosphorylates Ser43 in Yotiao to enhance cAMP-dependent activation of $I_{\mathrm{Ks}}$. Although the precise mechanism is not clear, it seems that a disruption of interactions between the AKAP and the KCNQ1 subunit might be responsible for the impaired $\beta$-adrenergic regulation of $I_{\mathrm{KS}}$ detected in some individuals with KCNQ1 mutations.

Second, Gravin (also known as AKAP250) is another anchoring protein that binds PKA and PKC. Work from Malbon and colleagues [53,54] suggests that Gravin is a substrate for its own associated kinases and that phosphorylation facilitates the association of this AKAP with agonist-occupied $\beta$-adrenergic receptors.
Third, AKAP-Lbc is a multifunctional PKA- and PKCanchoring protein that acts as a guanine nucleotide exchange factor (GEF) for the small GTPase Rho and synchronizes the activation of a third protein kinase called protein kinase D (PKD) [31,55,56]. Work published concurrently by the Diviani [57] and Pawson [58] groups has shown that PKA-mediated phosphorylation of Ser1565 in AKAP-Lbc generates a binding site for 14-33 proteins, which suppress the Rho-GEF activity of AKAPLbc (Figure 2b). The synergistic actions of anchored PKA

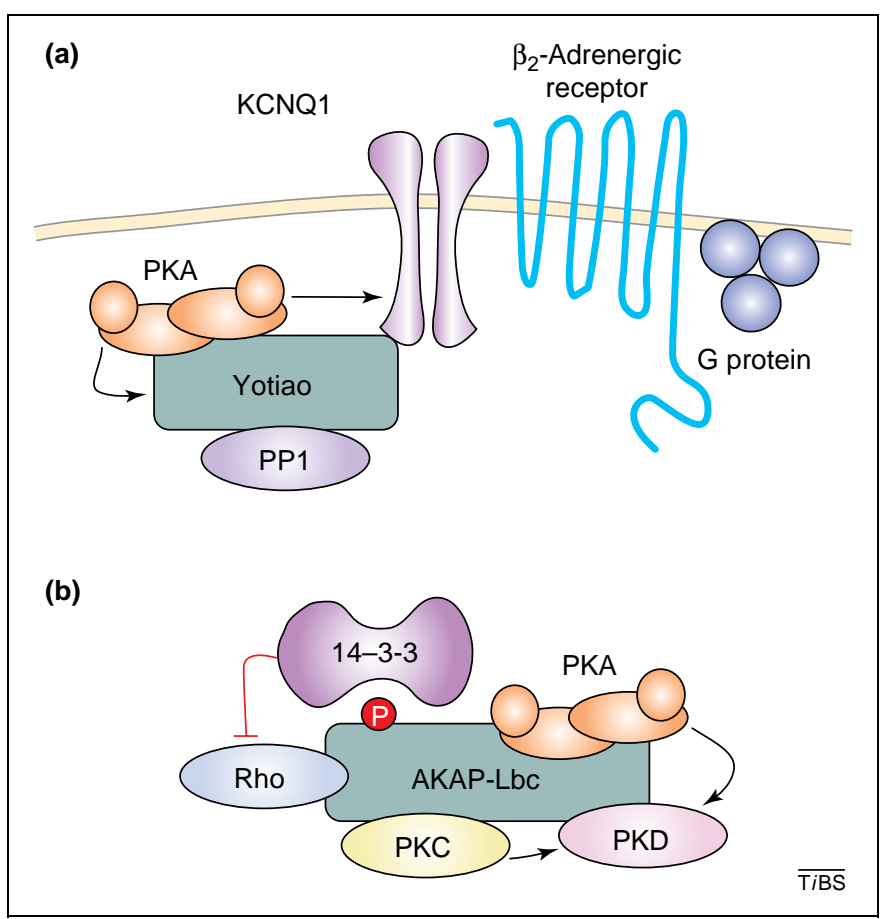

Figure 2. Phosphorylation of AKAPs regulates their function. (a) Yotiao couples PKA and $P P 1$ to KCNQ1 subunits that are part of the channel responsible for $I_{K S}$ currents. $\beta_{2}$-Adrenergic signaling activates PKA, which then phosphorylates the channel and Yotiao, leading to cAMP-dependent activation of the channel. Yotiao seems to act as both a scaffold for coordinating local phosphorylation or dephosphorylation events and an accessory subunit that shapes channel output. (b) AKAP-Lbc is a RhoGEF that also anchors PKA, PKC and PKD. Phosphorylation of AKAP-Lbc by anchored PKA regulates the activation of PKD via PKC and inhibits Rho-GEF activity by inducing $14-3-3$ binding. 
and PKC also contribute to activation of PKD. Phosphorylation by $\mathrm{PKC} \eta$ primes PKD for activation, whereas PKA-mediated phosphorylation of Ser2737 in AKAP-Lbc releases PKD from the activation complex [31] (Figure 2b).

These examples of highly localized phosphorylation events that occur in AKAP signaling complexes highlight the utility of kinase anchoring as a means to restrict the substrate accessibility of broad-spectrum enzymes such as PKA and PKC.

\section{Combinatorial assembly of distinct AKAP signaling complexes}

A basic premise of AKAP action is that signaling specificity is obtained when enzymes are targeted towards selected substrates. This use of distinct enzyme combinations provides a way in which to expand the repertoire of cellular events that can be modulated by a given AKAP. This concept is illustrated by work published in 2005 by Hoshi et al. [59] showing that AKAP79/150 coordinates different enzyme combinations to modulate the activity of two distinct neuronal ion channels: AMPA-type glutamate receptors and $\mathrm{KCNQ}_{2} \mathrm{~K}^{+}$channels (Figure 3a,b) [59]. These studies investigated the mechanism of agonist-induced rundown of AMPA currents, a process that is known to involve AKAP79/150 (Figure 3a). PKA-mediated phosphorylation of the AMPA channel stabilizes the current [60]; this process is opposed by PP2B-mediated dephosphorylation of the channel [61].

Using a new technique combining RNA interference of the endogenous protein in neurons and replacement with AKAP79/150 forms unable to anchor selected binding partners, Hoshi et al. [59] have shown that PP2B anchoring is primarily responsible for AMPA channel rundown. The same strategy applied to cervical ganglion neurons (SCGs) demonstrated that AKAP79/150 and PKC are involved in modulating $\mathrm{M}$ current $\left(\mathrm{a} \mathrm{K}^{+}\right.$conductance that negatively regulates neuronal excitability) [59]. These data point to a fascinating situation. In hippocampal neurons, AKAP79/150 coordinates PKA- and PP2B-mediated modulation of AMPA currents, but any AKAP79/150-associated PKC remains inactive in this process. By contrast, AKAP79/150 enables PKC to facilitate M-current regulation in SCG neurons, whereas PKA and PP2B seem to be non-essential (Figure 3b).
Interestingly, AKAP79/150 can also bind to $\beta$-adrenergic receptors [62-64]. Potentially, this binding permits the assembly of a third AKAP79/150 signaling complex in which PKA contributes to phosphorylation-dependent downregulation of the $\beta$-adrenergic receptors (Figure 3c).

Although the contextual cues that drive the preferential assembly of these three different AKAP79/150 complexes are unclear, one possibility is that the initial binding event between the anchoring protein and its target substrate promotes a sequence of conformational changes that directs recruitment of the next binding partners. For example, the association of AKAP79/150 with the KCNQ2 channel might provide a configuration that retains the membrane tethering and anchoring of PKC. Similarly, the formation of a ternary complex containing AMPA channels, the MAGUK adaptor protein and AKAP79/150 might have to be established before PKA and PP2B can be orientated towards the channel. Other factors, however, such as the co-translational assembly of protein complexes via localized protein synthesis, or species-specific or cell-type-specific expression of particular binding partners might also influence the composition of these 'context-dependent' signaling networks [65] (Figure 3).

Another way in which to vary the 'context-dependent' modulation of signaling networks might be to induce timedependent changes in AKAP complexes. For example, pathophysiological changes in the mAKAP complex have been recently linked to some forms of heart failure. Ryanodine receptors (RyRs) are intracellular $\mathrm{Ca}^{2+}$ release channels that are present in multiprotein signaling complexes at the sarcoplasmic reticulum in muscle cells to mediate excitation-contraction coupling. Upon $\beta$-adrenergic stimulation, anchored PKA phosphorylates the RyR to sensitize the channel to activation by an increase in $\mathrm{Ca}^{2+}$. Much work suggests that chronic changes in the mAKAP-RyR1 complex, including loss of anchored PDE4D3 and hyperactivation of the anchored PKA, correlate with the onset of 'leaky' channels found in some models of exercise-induced cardiac arrhythmias and heart failure [66,67]. Whether these changes in the mAKAP complex can be detected routinely in individuals affected with some types of heart disease, or whether this protein represents a viable therapeutic target for (a)

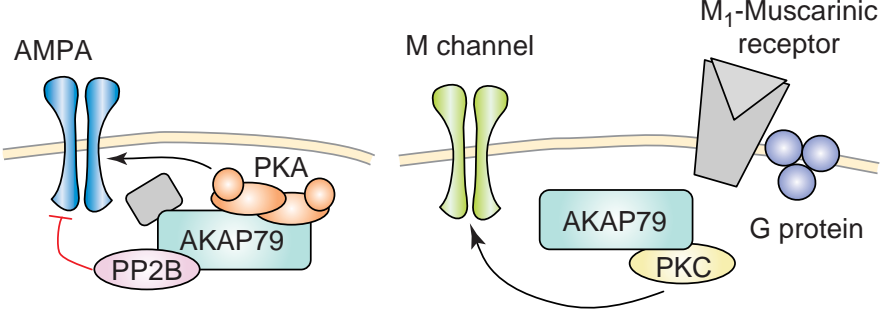

(c)

$\beta$-Adrenergic

receptor

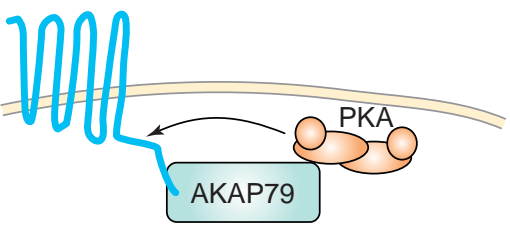

Figure 3. Combinatorial assembly of distinct enzyme complexes on AKAP79. This AKAP is coupled to AMPA glutamate receptors, KCNQ2 channel complexes and $\beta$ -

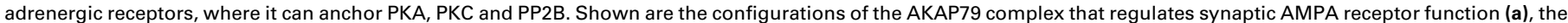

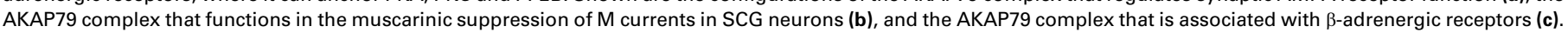
Different combinations of AKAP79-binding partners are used with each substrate. 
(a) 1991: dye-labeled cAMP reporter; intermolecular FRET

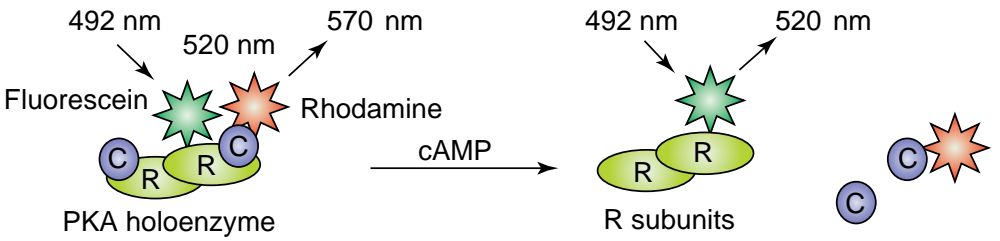

(b) 2001: mutant CNG channel coupled with Fura-2
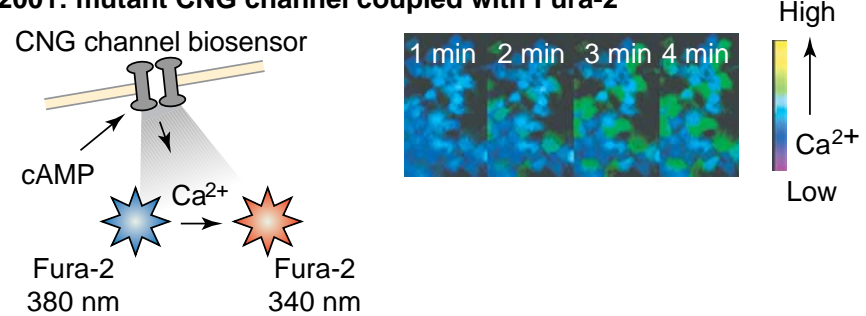

$380 \mathrm{~nm} \quad 340 \mathrm{~nm}$

(c) 2002: genetically encoded cAMP reporter; intermolecular FRET

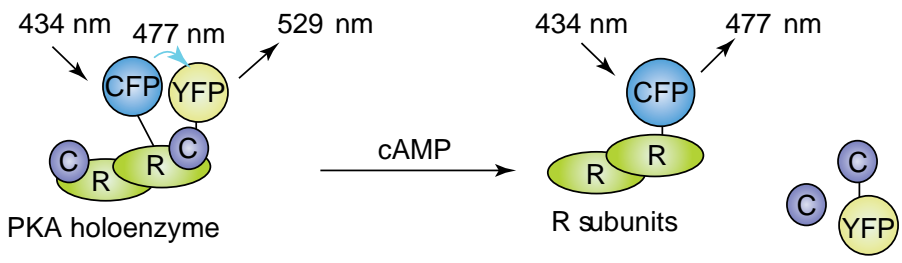

(d) 2004: ICUE1 indicator of cAMP; intramolecular FRET

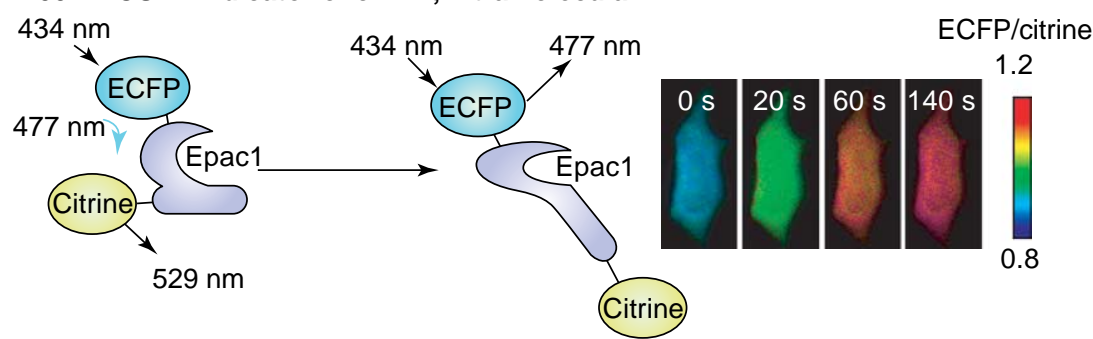

(e) 2005: AKAR2 PKA activity reporter; intramolecular FRET

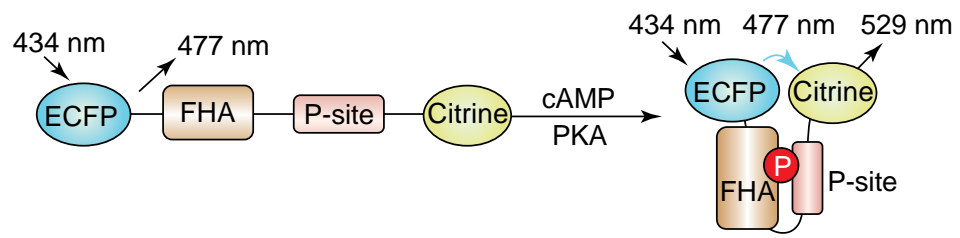

AKAR-PKA (PKA targeting/PKA activity reporter)
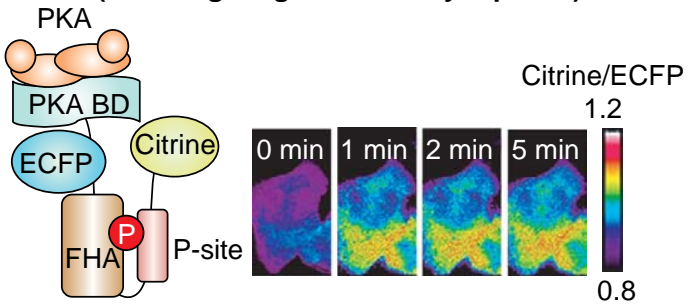

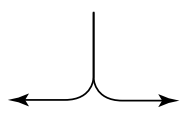

AKAR-PKA-PDE

(PKA/PDE4D3 targeting/activity reporter)

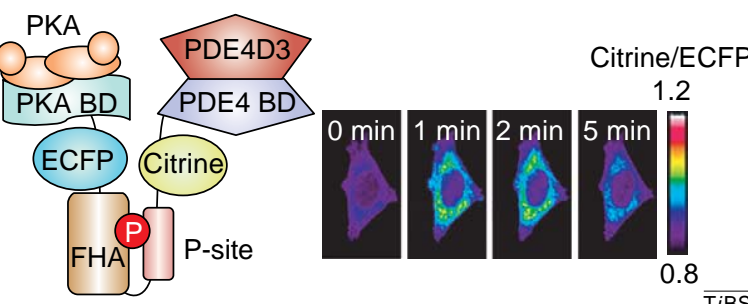

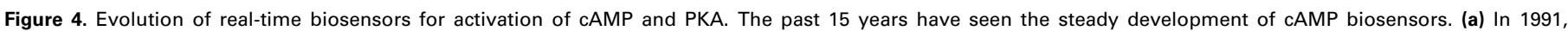
fluorescently labeled regulatory (R) and catalytic (C) subunits were microinjected into cells for monitoring intermolecular FRET. (b) By 2001, activation of cAMP could be

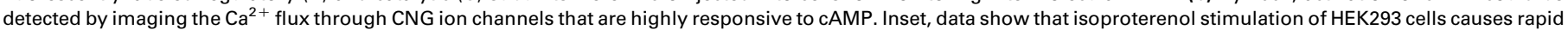

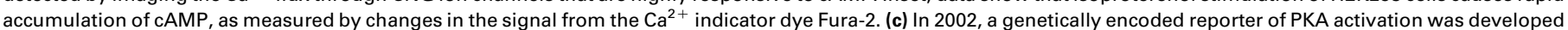

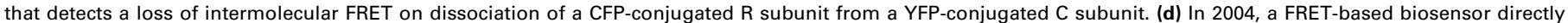

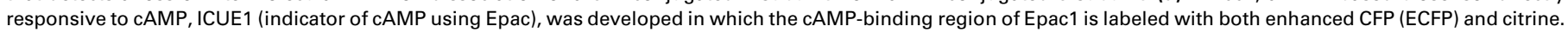

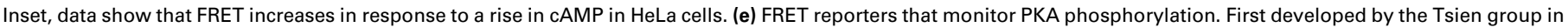


intervention, remains to be seen. Nonetheless, these findings yet again support an active role for AKAPs in synchronization of physiologically relevant signaling events.

\section{Time: the final frontier}

Although we have identified most, if not all, of the proteins that make up the cAMP signal transduction cascade, we still face the challenge of resolving the mechanics of their action in real time. Fluorescent probes that report the activation dynamics of cAMP effector proteins such as PKA, cyclic-nucleotide-gated (CNG) ion channels and Epac-GEFs have become the methods of choice to visualize the dynamics of cAMP signaling inside cells. Pioneering work by Roger Tsien's laboratory (http://www.tsienlab. ucsd.edu/) led to the development of a PKA-based probe that could monitor cAMP production by the loss of fluorescence resonance energy transfer (FRET) between recombinant regulatory (R) and catalytic (C) subunits conjugated with fluorescein and rhodamine dyes, respectively [68] (Figure 4a).

A decade later, Zaccolo and Pozzan [69] improved this technique by creating a genetically encoded PKA reporter that recorded FRET between a yellow fluorescent protein (YFP)-conjugated $\mathrm{C}$ subunit and a cyan fluorescent protein (CFP)-conjugated $R$ subunit (Figure $4 b$ ). This reporter was successfully used to measure microdomains of cAMP along sarcomeric $\mathrm{Z}$ lines in cardiomyocytes in response to adrenergic stimulation. More recently, Zaccolo, Pozzan and co-workers [70] have shown that AKAPs are involved in anchoring the kinase to these regions and that compartmentalized pools of PDE3 and PDE4 suppress signals located in these cAMP microdomains.

Other investigators have created biosensors based on the properties of CNG channels and Epacs. $\mathrm{Ca}^{2+}$ influx through CNG ion channels is stimulated in response to an increase in cAMP. When coupled with the $\mathrm{Ca}^{2+}$ sensitive indicator Fura-2 and PDE inhibitors, mutants of cAMP-selective CNG channels function as real-time biosensors of cAMP accumulation at the plasma membrane [71-73] (Figure 4c). Likewise, the cAMPbinding domain of the Epac-GEF has been used to generate fluorescent cAMP biosensors that detect compartmentalized accumulation of the second messenger [74,75] (Figure 4d).

FRET-based reporters of PKA activity provide an alternative to measuring cAMP concentrations. AKAR2 is a chimeric protein consisting of CFP, a consensus PKA substrate sequence, a Forkhead Homology (FHA) domain that binds phosphoamino acids, and the YFP variant citrine. PKA-mediated phosphorylation of the PKA consensus site engages the FHA domain to enhance FRET between the fluorescent moieties [76,77] (Figure 4e). This reporter also samples local phosphatase activity, because FRET decay is dependent on dephosphorylation of the PKA site and relaxation of the molecule. AKAR2 reporters have been recently used to examine two aspects of PKA activation dynamics.

First, chronic insulin treatment delays activation of PKA through $\beta$-adrenergic receptors in differentiated 3T3-L1 adipocytes. Work by Zhang et al. [76] suggests that in chronic hyperinsulinemia complexes of the $\beta$-adrenergic receptor and AKAP are decompartmentalized in relation to the cAMP synthesis machinery.

Second, Dodge-Kafka et al. [43] have used a modified AKAR2 reporter to show that recruitment of PKA and a PDE into the FRET reporter complex generates localized pulses of cAMP that are shorter in duration than when PDE is not present (Figures 4e). Thus, various cAMP-responsive events that have differing durations and are responsive to distinct thresholds of cAMP might emanate from the same microdomain. This possibility would be particularly relevant to mAKAP signaling complexes that contain three functionally distinct cAMP-dependent enzymes (PKA, PDE4D3 and Epac1): PKA is responsive to nanomolar concentrations of cAMP and would become active early in a second messenger response; by contrast, the activities of PDE4D3 (Michaelis constant, $K_{\mathrm{m}}=1-4 \mu \mathrm{M}$ ) and Epac1 (dissociation constant, $K_{\mathrm{d}}=4 \mu \mathrm{M}$ ) would commence only when cAMP accumulated to micromolar concentrations [43]. Conversely, inactivation of PDE4D3 and Epac1 would precede reformation of the PKA holoenzyme as the cAMP concentrations declined.

\section{Concluding remarks}

We anticipate that these cutting-edge imaging technologies will be central to obtaining precise definitions of the spatial and temporal patterns of anchored kinase function in the next decade. The information gained could be used in conjunction with computational models of signal transduction to predict the effects of perturbing the system. Ultimately, a balance of both approaches might contribute to the development of therapeutics that target 'signaling diseases' such as heart failure, asthma, diabetes and cancer. Therefore, pharmacological manipulation of kinase signaling in space and time will ultimately give us more control over where and when things happen in the cell.

\section{Acknowledgements}

We thank Debbie Willoughby, Dermot Cooper and Jin Zhang for providing unpublished data for use in Figure 4; Joe Soughayer for the FRET images in Figure 4; and all members of the Scott Laboratory for helpful discussions. J.D.S. was supported in part by a grant from the National Institutes of Health (DK54441).

2001 [77], the AKAR reporters use phosphorylation-dependent intramolecular interactions as an index of PKA activation. More recent versions of this reporter contain the

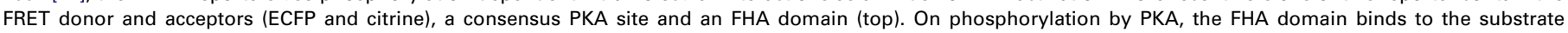
phosphothreonine and FRET increases. This reporter has been modified to study signaling by anchored PKA and the role of PDEs by incorporating an RII-binding peptide

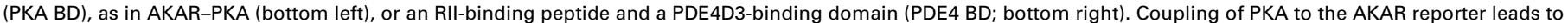
long-lasting PKA activity and FRET (left). The duration of this signal is attenuated when PDE4D3 is bound to the reporter (right). 


\section{References}

1 Fischer, E.H. and Krebs, E.G. (1955) Conversion of phosphorylase $b$ to phosphorylase $a$ in muscle extracts. J. Biol. Chem. 216, 121-132

2 Krebs, E.G. et al. (1959) Factors affecting the activity of muscle phosphorylase $b$ kinase. J. Biol. Chem. 234, 2867-2873

3 Eckhart, W. et al. (1979) An activity phosphorylating tyrosine in polyoma $\mathrm{T}$ antigen immunoprecipitates. Cell 18, 925-933

4 Brugge, J.S. et al. (1979) Detection of the viral sarcoma gene product in cells infected with various strains of avian sarcoma virus and of a related protein in uninfected chicken cells. J. Virol. 29, 1196-1203

5 Sadowski, I. et al. (1986) A non-catalytic domain conserved among cytoplasmic protein-tyrosine kinases modifies the kinase function and transforming activity of Fujinami sarcoma virus P130gag-fps. Mol. Cell. Biol. 6, 4396-4408

6 Knighton, D.R. et al. (1991) Structure of a peptide inhibitor bound to the catalytic subunit of cyclic adenosine monophosphate-dependent protein kinase. Science 253, 414-420

7 Manning, G. et al. (2002) The protein kinase complement of the human genome. Science 298, 1912-1934

8 Buxton, I.L.O. and Brunton, L.L. (1983) Compartments of cyclic AMP and protein kinase in mammalian cardiomyocytes. J. Biol. Chem. 258, 10233-10239

9 Theurkauf, W.E. and Vallee, R.B. (1982) Molecular characterization of the cAMP-dependent protein kinase bound to microtubule-associated protein 2. J. Biol. Chem. 257, 3284-3290

10 Lohmann, S.M. et al. (1984) High-affinity binding of the regulatory subunit (RII) of cAMP-dependent protein kinase to microtubuleassociated and other cellular proteins. Proc. Natl. Acad. Sci. U. S. A. 81, 6723-6727

11 Leiser, M. et al. (1986) Differential binding of the regulatory subunits (RII) of cAMP-dependent protein kinase II from bovine brain and muscle to RII-binding proteins. J. Biol. Chem. 261, 1904-1908

12 Bregman, D.B. et al. (1989) High affinity binding protein for the regulatory subunit of cAMP-dependent protein kinase II-B. Cloning, characterization, and expression of cDNAs for rat brain P150. J. Biol. Chem. 264, 4648-4656

13 Scott, J.D. et al. (1990) Type II regulatory subunit dimerization determines the subcellular localization of the cAMP-dependent protein kinase. J. Biol. Chem. 265, 21561-21566

14 Carr, D.W. et al. (1991) Interaction of the regulatory subunit (RII) of cAMP-dependent protein kinase with RII-anchoring proteins occurs through an amphipathic helix binding motif. J. Biol. Chem. 266, 14188-14192

15 Carr, D.W. and Scott, J.D. (1992) Blotting and band-shifting: techniques for studying protein-protein interactions. Trends Biochem. Sci. 17, 246-249

16 Carr, D.W. et al. (1992) Localization of the cAMP-dependent protein kinase to the postsynaptic densities by A-kinase anchoring proteins: characterization of AKAP79. J. Biol. Chem. 267, 16816-16823

17 Carr, D.W. et al. (1992) Association of the type II cAMP-dependent protein kinase with a human thyroid RII-anchoring protein. Cloning and characterization of the RII-binding domain. J. Biol. Chem. 267, 13376-13382

18 Rosenmund, C. et al. (1994) Anchoring of protein kinase A is required for modulation of AMPA/kainate receptors on hippocampal neurons. Nature 368, 853-856

19 Johnson, B.D. et al. (1994) Voltage-dependent potentiation of L-type $\mathrm{Ca}^{2+}$ channels in skeletal muscle cells requires anchored cAMPdependent protein kinase. Proc. Natl. Acad. Sci. U. S. A. 91, 11492-11496

20 Vijayaraghavan, S. et al. (1997) Protein kinase A-anchoring inhibitor peptides arrest mammalian sperm motility. J. Biol. Chem. 272, $4747-4752$

21 Burns-Hamuro, L.L. et al. (2003) Designing isoform-specific peptide disruptors of protein kinase A localization. Proc. Natl. Acad. Sci. U. S. A. $100,4072-4077$

22 Alto, N.M. et al. (2003) Bioinformatic design of A-kinase anchoring protein-in silico: a potent and selective peptide antagonist of type II protein kinase A anchoring. Proc. Natl. Acad. Sci. U. S. A. 100, $4445-4450$

23 Newlon, M.G. et al. (1999) The molecular basis for protein kinase A anchoring revealed by solution NMR. Nat. Struct. Biol. 6, 222-227
24 Newlon, M.G. et al. (2001) A novel mechanism of PKA anchoring revealed by solution structures of anchoring complexes. EMBO J. 20, $1651-1662$

25 Huang, L.J. et al. (1997) Identification of a novel dual specificity protein kinase A anchoring protein, D-AKAP1. J. Biol. Chem. 272, 8057-8064

26 Huang, L.J. et al. (1997) D-AKAP2, a novel protein kinase A anchoring protein with a putative RGS domain. Proc. Natl. Acad. Sci. U. S. A. 94, 11184-11189

27 Tasken, K. and Aandahl, E.M. (2004) Localized effects of cAMP mediated by distinct routes of protein kinase A. Physiol. Rev. 84, 137-167

28 Coghlan, V.M. et al. (1995) Association of protein kinase A and protein phosphatase 2B with a common anchoring protein. Science 267, 108-112

29 Klauck, T.M. et al. (1996) Coordination of three signaling enzymes by AKAP79, a mammalian scaffold protein. Science 271, 1589-1592

30 Nauert, J.B. et al. (1997) Gravin, an autoantigen recognized by serum from myasthenia gravis patients, is a kinase scaffold protein. Curr. Biol. 7, 52-62

31 Carnegie, G.K. et al. (2004) AKAP-Lbc nucleates a protein kinase D activation scaffold. Mol. Cell 15, 889-899

32 Schillace, R.V. and Scott, J.D. (1999) Association of the type 1 protein phosphatase PP1 with the A-kinase anchoring protein AKAP220. Curr. Biol. 9, 321-324

33 Steen, R.L. et al. (2000) Recruitment of protein phosphatase 1 to the nuclear envelope by A-kinase anchoring protein AKAP149 is a prerequisite for nuclear lamina assembly. J. Cell Biol. 150, 1251-1262

34 Houslay, M.D. and Adams, D.R. (2003) PDE4 cAMP phosphodiesterases: modular enzymes that orchestrate signalling cross-talk, desensitization and compartmentalization. Biochem. J. 370, 1-18

35 Yarwood, S.J. et al. (1999) The RACK1 signaling scaffold protein selectively interacts with the cAMP-specific phosphodiesterase PDE4D5 isoform. J. Biol. Chem. 274, 14909-14917

36 Perry, S.J. et al. (2002) Targeting of cyclic AMP degradation to $\beta 2$ adrenergic receptors by $\beta$-arrestins. Science $298,834-836$

37 Verde, I. et al. (2001) Myomegalin is a novel protein of the Golgi/centrosome that interacts with a cyclic nucleotide phosphodiesterase. J. Biol. Chem. 276, 11189-11198

38 Dodge, K.L. et al. (2001) mAKAP assembles a protein kinase A/PDE4 phosphodiesterase cAMP signaling module. EMBO J. 20, 1921-1930

39 Baillie, G.S. et al. (2005) Compartmentalisation of phosphodiesterases and protein kinase A: opposites attract. FEBS Lett. 579, 3264-3270

40 Carlisle Michel, J.J. et al. (2004) PKA phosphorylation of PDE4D3 facilitates recruitment of the mAKAP signaling complex. Biochem. J. $381,587-592$

41 Sette, C. and Conti, M. (1996) Phosphorylation and activation of a cAMP-specific phosphodiesterase by the cAMP-dependent protein kinase. J. Biol. Chem. 271, 16526-16534

42 Hoffmann, R. et al. (1999) The MAP kinase ERK2 inhibits the cyclic AMP-specific phosphodiesterase HSPDE4D3 by phosphorylating it at Ser579. EMBO J. 18, 893-903

43 Dodge-Kafka, K.L. et al. (2005) The protein kinase A anchoring protein mAKAP coordinates two integrated cAMP effector pathways. Nature 437, 574-578

44 Michel, J.J. et al. (2005) Spatial restriction of PDK1 activation cascades by anchoring to mAKAP $\alpha$. Mol. Cell 20, 661-672

45 Tasken, K.A. et al. (2001) Phosphodiesterase 4D and protein kinase a type II constitute a signaling unit in the centrosomal area. J. Biol. Chem. 276, 21999-22002

46 Asirvatham, A.L. et al. (2004) A-kinase anchoring proteins interact with phosphodiesterases in T lymphocyte cell lines. J. Immunol. 173, 4806-4814

47 Bajpai, M. et al. (2006) AKAP3 selectively binds PDE4A isoforms in bovine spermatozoa. Biol. Reprod. 74, 109-118

48 Coghlan, V.M. et al. (1993) A-kinase anchoring proteins: a key to selective activation of cAMP-responsive events? Mol. Cell. Biochem. 127, 309-319

49 Westphal, R.S. et al. (1999) Regulation of NMDA receptors by an associated phosphatase-kinase signaling complex. Science 285, 93-96

50 Kass, R.S. and Moss, A.J. (2003) Long QT syndrome: novel insights into the mechanisms of cardiac arrhythmias. J. Clin. Invest. 112, $810-815$ 
51 Marx, S.O. et al. (2002) Requirement of a macromolecular signaling complex for $\beta$ adrenergic receptor modulation of the KCNQ1-KCNE1 potassium channel. Science $295,496-499$

52 Chen, L. et al. (2005) Phosphorylation of the A-kinase-anchoring protein Yotiao contributes to protein kinase A regulation of a heart potassium channel. J. Biol. Chem. 280, 31347-31352

53 Shih, M. et al. (1999) Dynamic complexes of $\beta 2$-adrenergic receptors with protein kinases and phosphatases and the role of gravin. J. Biol. Chem. 274, 1588-1595

54 Tao, J. et al. (2003) Protein kinase A regulates AKAP250 (gravin) scaffold binding to the $\beta 2$-adrenergic receptor. $E M B O \quad J .22$, 6419-6429

55 Diviani, D. et al. (2001) AKAP-Lbc anchors protein kinase A and nucleates G $\alpha 12$-selective Rho-mediated stress fiber formation. J. Biol. Chem. 276, 44247-44257

56 Klussmann, E. et al. (2001) Ht31: the first protein kinase A anchoring protein to integrate protein kinase A and Rho signaling. FEBS Lett. 507, 264-268

57 Diviani, D. et al. (2004) Anchoring of both PKA and 14-3-3 inhibits the Rho-GEF activity of the AKAP-Lbc signaling complex. EMBO J. 23, $2811-2820$

58 Jin, J. et al. (2004) Proteomic, functional, and domain-based analysis of in vivo 14-3-3 binding proteins involved in cytoskeletal regulation and cellular organization. Curr. Biol. 14, 1436-1450

59 Hoshi, N. et al. (2005) Distinct enzyme combinations in AKAP signalling complexes permit functional diversity. Nat. Cell Biol. 7, 1066-1073

60 Banke, T.G. et al. (2000) Control of GluR1 AMPA receptor function by cAMP-dependent protein kinase. J. Neurosci. 20, 89-102

61 Tavalin, S.J. et al. (2002) Regulation of GluR1 by the A-kinase anchoring protein 79 (AKAP79) signaling complex shares properties with long-term depression. J. Neurosci. 22, 3044-3051

62 Fraser, I.D. et al. (2000) Assembly of an A kinase-anchoring protein$\beta_{2}$-adrenergic receptor complex facilitates receptor phosphorylation and signaling. Curr. Biol. 10, 409-412

63 Cong, M. et al. (2001) Regulation of membrane targeting of the G protein-coupled receptor kinase 2 by protein kinase $\mathrm{A}$ and its anchoring protein AKAP79. J. Biol. Chem. 276, 15192-15199

64 Lynch, M.J. et al. (2005) RNA silencing identifies PDE4D5 as the functionally relevant cAMP phosphodiesterase interacting with $\beta$ arrestin to control the protein kinase A/AKAP79-mediated switching of the 32 -adrenergic receptor to activation of ERK in HEK293B2 cells. J. Biol. Chem. 280, 33178-33189

65 Chen, L. and Kass, R.S. (2005) A-kinase anchoring proteins: different partners, different dance. Nat. Cell Biol. 7, 1050-1051

66 Marx, S.O. et al. (2001) Phosphorylation-dependent regulation of ryanodine receptors: a novel role for leucine/isoleucine zippers. J. Cell Biol. 153, 699-708

67 Lehnart, S.E. et al. (2005) Phosphodiesterase 4D deficiency in the ryanodine-receptor complex promotes heart failure and arrhythmias. Cell 123, 25-35

68 Adams, S.R. et al. (1991) Fluorescence ratio imaging of cyclic AMP in single cells. Nature 349, 694-697

69 Zaccolo, M. and Pozzan, T. (2002) Discrete microdomains with high concentration of cAMP in stimulated rat neonatal cardiac myocytes. Science 295, 1711-1715

70 Mongillo, M. et al. (2004) Fluorescence resonance energy transferbased analysis of cAMP dynamics in live neonatal rat cardiac myocytes reveals distinct functions of compartmentalized phosphodiesterases. Circ. Res. 95, 67-75

71 Fagan, K.A. et al. (1999) Adenovirus-mediated expression of an olfactory cyclic nucleotide-gated channel regulates the endogenous $\mathrm{Ca}^{2+}$-inhibitable adenylyl cyclase in C6-2B glioma cells. J. Biol. Chem. 274, 12445-12453

72 Rich, T.C. et al. (2000) Cyclic nucleotide-gated channels colocalize with adenylyl cyclase in regions of restricted cAMP diffusion. J. Gen. Physiol. 116, 147-161

73 Rich, T.C. et al. (2001) In vivo assessment of local phosphodiesterase activity using tailored cyclic nucleotide-gated channels as cAMP sensors. J. Gen. Physiol. 118, 63-78

74 Nikolaev, V.O. et al. (2004) Novel single chain cAMP sensors for receptor-induced signal propagation. J. Biol. Chem. 279, 37215-37218

75 DiPilato, L.M. et al. (2004) Fluorescent indicators of cAMP and Epac activation reveal differential dynamics of cAMP signaling within discrete subcellular compartments. Proc. Natl Acad. Sci. U. S. A. 101, 16513-16518

76 Zhang, J. et al. (2005) Insulin disrupts $\beta$-adrenergic signalling to protein kinase A in adipocytes. Nature 437, 569-573

77 Zhang, J. et al. (2001) Genetically encoded reporters of protein kinase A activity reveal impact of substrate tethering. Proc. Natl Acad. Sci. U. S. A. $98,14997-15002$

\section{AGORA initiative provides free agriculture journals to developing countries}

The Health Internetwork Access to Research Initiative (HINARI) of the WHO has launched a new community scheme with the UN Food and Agriculture Organization.

As part of this enterprise, Elsevier has given 185 journals to Access to Global Online Research in Agriculture (AGORA). More than 100 institutions are now registered for the scheme, which aims to provide developing countries with free access to vital research that will ultimately help increase crop yields and encourage agricultural self-sufficiency.

According to the Africa University in Zimbabwe, AGORA has been welcomed by both students and staff. 'It has brought a wealth of information to our fingertips' says Vimbai Hungwe. 'The information made available goes a long way in helping the learning, teaching and research activities within the University. Given the economic hardships we are going through, it couldn't have come at a better time.'

For more information visit:

http://www.healthinternetwork.net 